\title{
On experimental determination of the random-incidence response of microphones
}

\author{
Barrera Figueroa, Salvador; Rasmussen, Knud; Jacobsen, Finn
}

Published in:

Journal of the Acoustical Society of America

Link to article, DOI:

$10.1121 / 1.2715655$

Publication date:

2007

Document Version

Publisher's PDF, also known as Version of record

Link back to DTU Orbit

Citation $(A P A)$ :

Barrera Figueroa, S., Rasmussen, K., \& Jacobsen, F. (2007). On experimental determination of the randomincidence response of microphones. Journal of the Acoustical Society of America, 121(5), 2628-2636. https://doi.org/10.1121/1.2715655

\section{General rights}

Copyright and moral rights for the publications made accessible in the public portal are retained by the authors and/or other copyright owners and it is a condition of accessing publications that users recognise and abide by the legal requirements associated with these rights.

- Users may download and print one copy of any publication from the public portal for the purpose of private study or research.

- You may not further distribute the material or use it for any profit-making activity or commercial gain

- You may freely distribute the URL identifying the publication in the public portal 


\title{
On experimental determination of the random-incidence response of microphones
}

\author{
Salvador Barrera-Figueroa ${ }^{\text {a) }}$ and Knud Rasmussen ${ }^{\text {b) }}$ \\ Danish Primary Laboratory of Acoustics - Danish Fundamental Metrology, Matematiktorvet B307, \\ DK-2800 Lyngby, Denmark \\ Finn Jacobsen ${ }^{\text {c) }}$ \\ Acoustic Technology - Ørsted-DTU, Technical University of Denmark, Ørsteds Plads B352, \\ DK-2800 Lyngby, Denmark
}

(Received 9 November 2006; revised 16 February 2007; accepted 17 February 2007)

\begin{abstract}
The random-incidence sensitivity of a microphone is defined as the ratio of the output voltage to the sound pressure that would exist at the position of the acoustic center of the microphone in the absence of the microphone in a sound field with incident plane waves coming from all directions. The random-incidence correction of a number of laboratory standard microphones has been determined experimentally. Although the measurement procedure seems to be straightforward, some practical and fundamental problems arise: (i) Reflections from the mounting rig contaminate the measured frequency response, and whereas some of these reflections can be removed using a time-selective technique, others coincide with the direct impulse response and consequently cannot be removed in the time domain and thus affect the accuracy of the estimate; (ii) the accuracy of the estimate is relying on the rotational symmetry of the microphone and depends on the angular resolution. The effect of the angular resolution has been compared with the analytical solution of the scattering and diffraction around a solid sphere. Numerical calculations supplement the experimental results. Although the procedure has only been applied to laboratory standard microphones, it is not restricted to such microphones and may be applied to other types of measurement microphones. (c) 2007 Acoustical Society of America. [DOI: 10.1121/1.2715655]
\end{abstract}

PACS number(s): 43.38.Kb, 43.58.Vb [AJZ]

Pages: 2628-2636

\section{INTRODUCTION}

The sensitivity of a microphone is defined as the ratio of the output voltage to the sound pressure that would exist at the position of the acoustic center of the microphone in the absence of the microphone in a specified sound field. During a calibration, the microphone can be subjected to a uniform sound pressure over the diaphragm, a plane wave in a free field, or a diffuse field. The first two cases have been extensively studied. These activities have led to the development of a number of standards ${ }^{1-3}$ while further investigations continue. $^{4-6}$ The random-incidence sensitivity has always been considered equivalent to the diffuse-field sensitivity, and used widely in practical applications. The randomincidence sensitivity is defined in terms of the assembled response of the microphone to plane waves from all possible directions impinging successively onto the microphone. ${ }^{7}$ However, determining the absolute sensitivity of a microphone at oblique angles of incidence is a very difficult process. Thus, instead of using the free-field sensitivities at each angle of incidence, a practical approach that allows the use of a relative response, such as the directivity factor or the directivity index, can be used with advantage.

The directivity factor can be determined using ${ }^{8}$

\footnotetext{
${ }^{a)}$ Electronic mail: sbf@dfm.dtu.dk

${ }^{b)}$ Electronic mail: kr@oersted.dtu.dk

${ }^{c)}$ Electronic mail: fja@oersted.dtu.dk
}

$$
Q(f)=4 \pi\left[\int_{\Omega}|G(\theta, \phi)|^{2} d \Omega\right]^{-1},
$$

where $f$ is the frequency, $\Omega$ is the solid angle $(4 \pi)$, and $G(\theta, \phi)$ is the ratio of the frequency response at the angles $\theta$ and $\phi$ to the frequency response at normal incidence, i.e., $G(\theta, \phi)=H(\theta, \phi) / H(0)$. If the microphone can be regarded as rotationally symmetrical, the dependence on $\phi$ disappears, and the directivity factor can be determined using the following expression: ${ }^{9}$

$$
Q(f)=2\left[\int_{0}^{\pi}|G(\theta)|^{2} \sin \theta d \theta\right]^{-1} .
$$

This expression can be used when an analytical form of the ratio of responses is available. However, this is not the case when the frequency response of a measurement microphone is determined experimentally. In such a case, the integral in Eq. (2) must be replaced by a discrete series,

$$
Q(f)=\frac{2\left|H\left(f, \theta_{0}\right)\right|^{2}}{\sum_{n=1}^{\pi / \Delta \theta}\left|H\left(f, \theta_{n}\right)\right|^{2} \sin \theta_{n} \Delta \theta} .
$$

Needless to say such a discretization is made under the assumption that it has no significant effect on the final accuracy of the estimated sensitivity. For example, a standard concerned with the determination of the diffuse field calibration of sound level meters specifies that each segment should not be larger than $3 \%$ of the total measurement area. ${ }^{7}$ The 
directivity index is the directivity factor in logarithmic form ${ }^{8}$

$$
\mathrm{DI}(f)=10 \log Q(f) .
$$

The directivity index can be used for determining the random-incidence sensitivity or the random-incidence correction. The random-incidence sensitivity is determined using

$$
M_{\mathrm{RI}}=M_{F, \theta=0}-\mathrm{DI}(f),
$$

where $M_{F, \theta=0}$ is the free-field sensitivity at normal incidence. The random-incidence correction can be calculated using

$$
C_{\mathrm{RI}}=C_{F, \theta=0}-\mathrm{DI}(f),
$$

where $C_{F, \theta=0}$ is the free-field correction at normal incidence. The free-field correction is the difference between the freefield sensitivity and the pressure sensitivity obtained either by reciprocity calibration or by calibrating using an electrostatic actuator. When a direct measurement of the randomincidence sensitivity is not available, it is common practice to use typical values of the random-incidence correction together with the pressure response of an individual microphone for determining the random-incidence sensitivity of the microphone.

The experimental determination of the directivity index is far from a simple and straightforward process. Measuring the frequency response at all incidence angles is not trivial. Two problems can be identified: (a) The measurements must be carried out in an anechoic environment, and (b) the error introduced by the use of the discrete angular positions around the microphone. Even in the best anechoic rooms the accuracy of the measurement will be degraded by reflections from the walls and from the measurement rig. Hence a technique that can remove such a negative effect is needed. A time-selective technique that has been developed for the reciprocity calibration of microphones in a free field can be applied with advantage. ${ }^{10}$

In this paper an exposition of the experimental determination of the directivity factor and the random-incidence correction of laboratory standard microphones is presented. The application of a time-selective technique for removing unwanted reflections from the frequency response is described. The effect of the angular resolution in the determination of the discrete directivity factor is studied and compared to the case of the scattering by a sphere. Numerical estimates obtained using the boundary element method (BEM) supplement the experimental results.

\section{EXPERIMENTAL SETUP}

The measurements required for determining the directivity index have to be made in a free field. Usually, this free field is realized in an anechoic room, but a simulated free field can also be used. Such a simulated free field is normally the result of time-selective techniques such as time delay spectrometry $^{11}$ or FFT-based techniques. ${ }^{10}$

\section{A. Measurement setup}

The measurement setup is composed of the measurement rig and the measurement instrumentation. The former

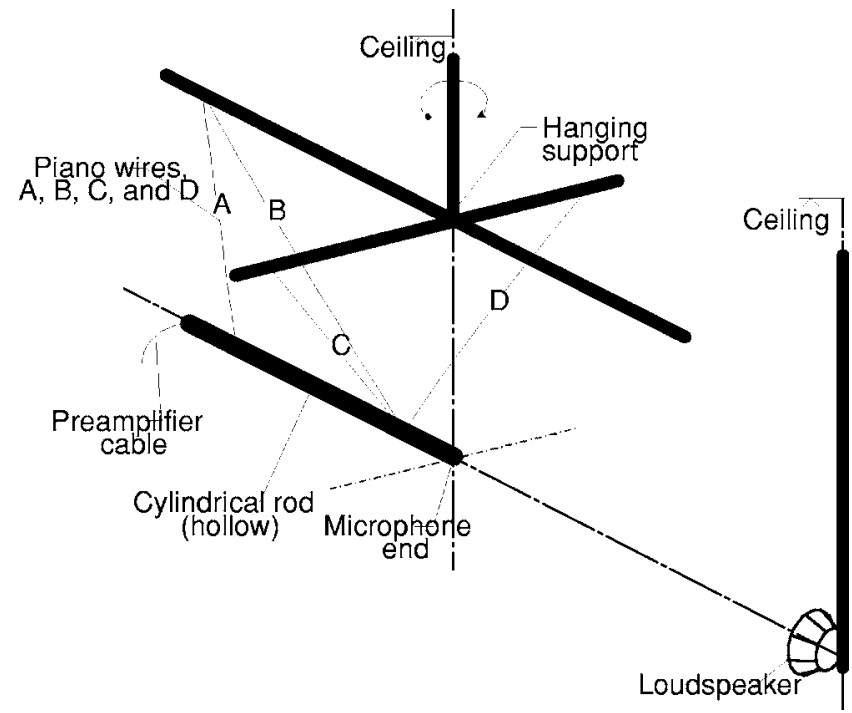

FIG. 1. Schematics of the mounting rig inside the anechoic room. The drawing is not to scale.

was mounted inside the anechoic room and the latter was placed in a control room. The measurement rig has to comply with two criteria: (i) It has to be as reflection-free as possible, and (ii) the rod where the microphones are mounted has to be long enough to be considered semi-infinite and it should have the same cross section as the microphone. A measurement rig was built complying with these two criteria. A scheme and a photograph of the measurement rig are shown in Figs. 1 and 2.

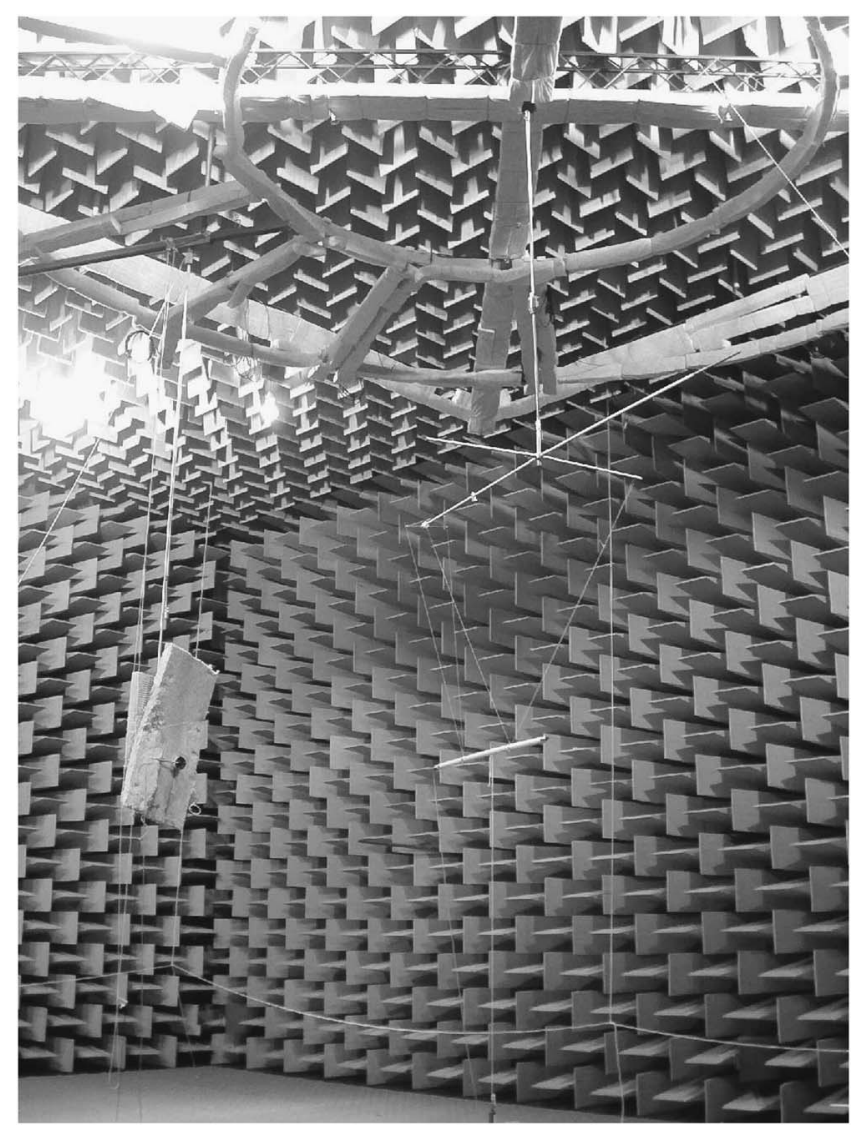

FIG. 2. Photograph of the experimental rig in the anechoic chamber. 


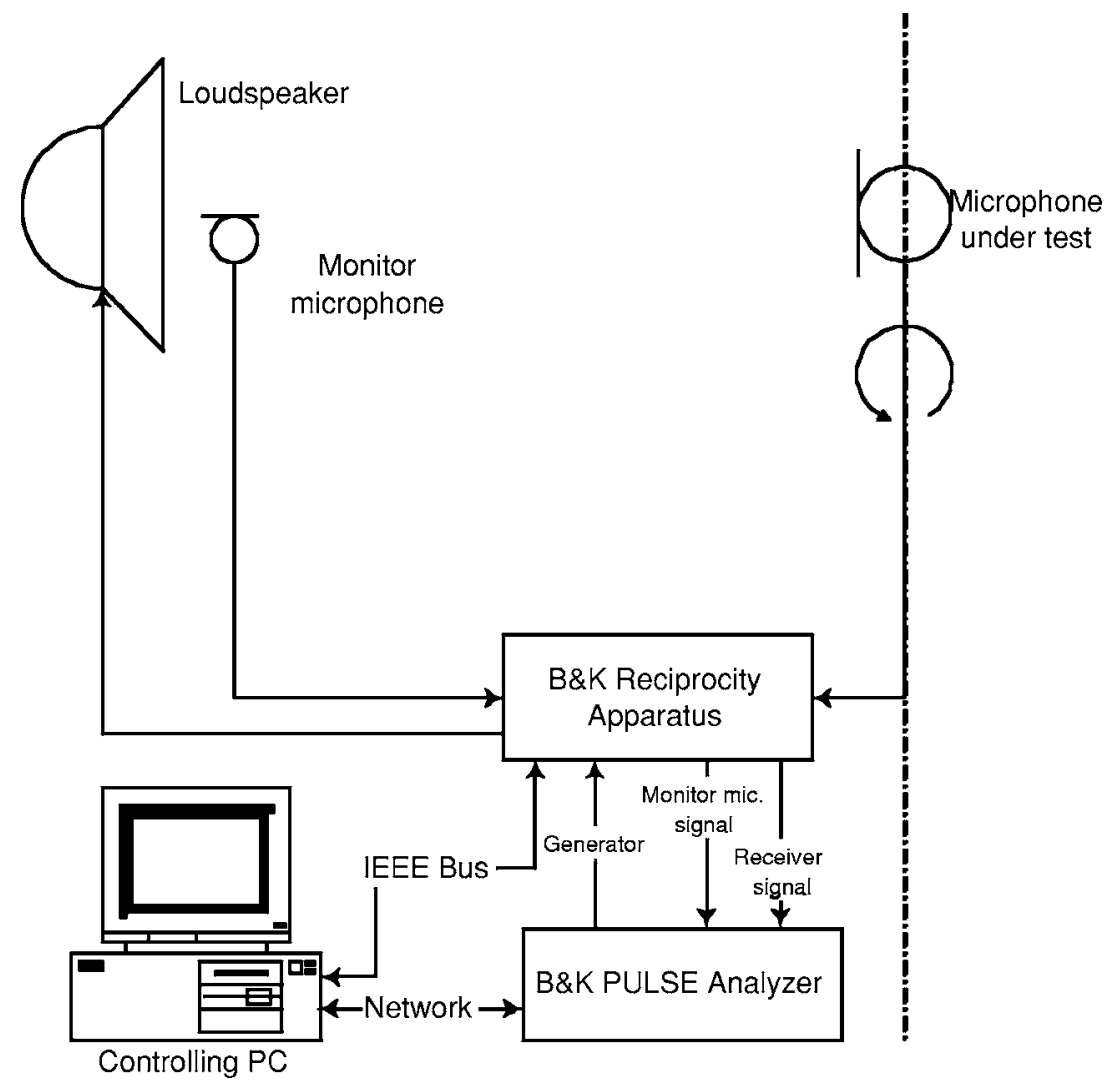

FIG. 3. Schematics of the measurement system used in the measurement of the directivity factor.
The rig is in the middle of a large anechoic room with free volume of nearly $1000 \mathrm{~m}^{3}$. The rod where the microphones are mounted is about $80 \mathrm{~cm}$ long, and it has a diameter of 23.77 or $12.7 \mathrm{~mm}$ depending on whether the microphones are 1-in. laboratory standard microphones (LS1) or $\frac{1}{2}$-in. laboratory standard microphones (LS2). The cross that hangs from the ceiling is about $1 \mathrm{~m}$ away from the microphone rod, and the piano wires are less than $1 \mathrm{~mm}$ in diameter. The microphone and microphone rod are aligned in such a way that the rig rotates around the diaphragm of the microphone. The loudspeaker is $1.7 \mathrm{~m}$ away from the microphone. A monitor microphone is placed in front of the loudspeaker.

The microphone under test and the monitor microphone are connected to a Brüel \& $\mathrm{Kjær}(\mathrm{B} \& \mathrm{~K})$ reciprocity apparatus model 5998. The output of the reciprocity apparatus is connected to a B\&K "PULSE" analyzer. The reciprocity apparatus is used for measuring the insert voltage in the channel of the microphone under test. The generator output of the PULSE analyzer is connected to a power amplifier that feeds the loudspeaker. The loudspeaker is a modified tweeter that has a flat frequency response up to $40 \mathrm{kHz}$. The monitor microphone is a $\frac{1}{4}$-in. condenser microphone, B\&K 4136. The analyzer and the reciprocity apparatus are connected to a PC that controls the measurements. Figure 3 shows the measurement system. Determining the frequency response of the test microphone at a given angle, $H(\theta)$, involves measuring the output voltage of the monitor and the test microphone. The measured frequency response is defined as the ratio of the open-circuit voltage of the microphone under test to the output voltage of the monitor microphone, $H_{\text {meas }}(f, \theta)$ $=U_{\text {test }}(f, \theta) / U_{\text {monitor }}(f)$. The frequency response is measured at discrete frequencies using stepped-sine excitation with $30 \mathrm{~Hz}$ step in the interval from 1 to $20 \mathrm{kHz}$ and from 2 to $30 \mathrm{kHz}$ for LS1 and LS2 microphones, respectively. Measurements below $1 \mathrm{kHz}$ were not made because of a high level of distortion of the loudspeaker.

\section{B. Removal of reflections from the rig}

The measurement rig has been carefully designed so as to minimize reflections that could contaminate the measured frequency response. However, many elements of the rig will reflect sound back to the microphones. In most cases these unwanted reflections could be removed using a timeselective technique. This technique has been tried before in free-field reciprocity calibrations. ${ }^{10}$

The procedure applied in this case was very similar: (a) The frequency response was measured as the transfer function between the signals of two microphones in a frequency interval $\left(f_{0}, f_{\max }\right)$; (b) the missing lower portion of the frequency response $\left(0, f_{0}\right)$ was calculated using theoretical data; (c) the high frequency response was taken smoothly to zero by applying a low-pass filter; (d) an inverse Fourier transform was calculated; (e) a time-selective window was applied to the resulting impulse response in order to remove reflections; and (f) a Fourier transform was applied to the "cleaned" impulse response.

If an inverse Fourier transform is to be applied onto the frequency response, this has to be defined in the whole frequency interval, i.e., from 0 to $\infty$ for a one-sided frequency response. The frequency response is completed following steps (b) and (c). Thus, the frequency response after step (b) can be defined as 


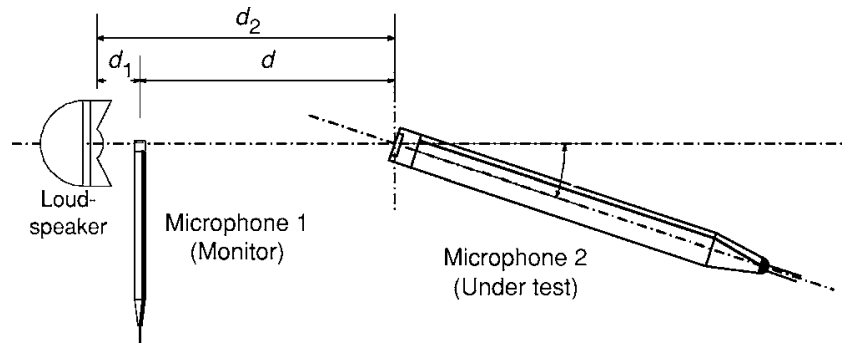

FIG. 4. Schematics of the relative positions of the microphones during the measurements (the drawing is not to scale).

$$
H_{\mathrm{c}}(f, \theta)=\left\{\begin{array}{l}
H^{\prime}(f, \theta), \quad 0 \leqslant f \leqslant f_{0} \\
H_{\text {meas }}(f, \theta), \quad f_{0} \leqslant f \leqslant f_{\text {max }},
\end{array}\right.
$$

where $H^{\prime}(f, \theta)$ can be estimated using the sensitivities and the distance between monitor and test microphones as follows:

$$
H^{\prime}(f, \theta)=\frac{p_{2} M_{F, 2}(f, \theta)}{p_{1} M_{F, 1}(f, \theta)},
$$

where $p_{1}$ and $p_{2}$ are the sound pressures at the position of microphones 1 and 2; and $M_{F, 1}(f, \theta)$, and $M_{F, 2}(f, \theta)$ and the free-field sensitivities of microphones 1 and 2 at the angle $\theta$ and the frequency $f$. Assuming that the source is a point source, and substituting the sound pressures at points 1 and 2 , this expression becomes

$$
H^{\prime}(f, \theta)=\frac{M_{F, 2}(f, \theta)}{M_{F, 1}(f, \theta)} \frac{d_{2}}{d_{2}+d} \exp (-i k d),
$$

where $d=d_{2}-d_{1}$ is the distance between microphones, $d_{1}$ and $d_{2}$ are the distances from the source to microphones 1 and 2, and $k$ is the wave number. In this case, microphone 1 was the monitor microphone, which remained at a fixed position in front of the loudspeaker while microphone 2, the microphone under test, was exposed to sound from a different angle of incidence at each measurement; a scheme is shown in Fig. 4. Thus, the resulting frequency response is basically the ratio of the sensitivities of the two microphones and the effect of the distance between the two microphones. Pressure sensitivities were used instead of the actual free-field sensitivities in Eq. (9), because at low frequencies there is little difference between the free-field sensitivity and the pressure sensitivity. In practice, the monitor microphone was located close to the source, and therefore interference caused by multiple reflections from the loudspeaker with the direct sound field may be expected. However, such effects are judged to be negligible in the frequency range of concern. In principle, the distance should take account of the positions of the acoustic centers of the microphones and the loudspeaker. ${ }^{7}$ However, as the distance between the microphone under test and the loudspeaker was about $1.7 \mathrm{~m}$, the effect of the acoustic center may be considered negligible.

The frequency response of a microphone will decay at high frequencies because of the fact that above the resonance frequency of the diaphragm, its movement is controlled by its mass, and thus the sensitivity of the microphone approaches zero. Therefore such a decay can be artificially made in the measured frequency range, provided that the upper frequency is at least two times the resonance frequency of the microphones. The decay can be accelerated by applying a low-pass filter. In this case, the low-pass filter was a linear phase finite impulse response (FIR) filter of 85th order, with a normalized passband frequency of 0.9 , and a stop-band attenuation of $80 \mathrm{~dB}$. The pass-band ripple was $0.0001 \mathrm{~dB}$. The frequency response after operation $(\mathrm{c})$ is

$$
H_{L}(f, \theta)=H_{c}(f, \theta) L(f),
$$

where $L(f)$ is the low-pass filter.

The impulse response was obtained after applying an inverse Fourier transform to $H_{\mathrm{L}}(f, \theta)$ [step (d)]. The timeselective window applied to the impulse response [step (e)] was a Tukey window with a duration of $3 \mathrm{~ms}$. The smoothing percentage of the sides of the window was $70 \%$. The window was long enough to eliminate all the reflections from the measurement rig, and the tapering reduces the ripple at the extremes of the frequency response. The length of the time selective window was defined from two criteria: (a) The distance between microphones, and (b) a visual inspection of the impulse response. The first criterion is based on the fact that the distance between microphones defines the instants when reflections from walls and other interferences occur. The second criterion is a visual inspection that ensures that no portion of the direct impulse response is excluded, and unwanted effects in as far as possible are excluded.

In some cases, the reflections could not be separated from the impulse response. This occurred at incidence angles larger than $120^{\circ}$. This is because perturbations from any element behind the microphone will be a part of the direct wave. Therefore, it is very important to minimize reflections coming from any element behind the microphone. For instance, the setup originally had a vertical rod that supported the horizontal rod where the microphone is located. The reflections from the vertical rod were significant, and they could not be removed from the measurement. Because of this, it was decided to modify the rig. The major change was the substitution of the vertical rod by piano wires. As can be seen in Fig. 5, only the reflection from the hanging preamplifier cable remained. This demonstrates a fundamental problem of the method: Unless the cable is removed, there is no easy way to get rid of such a reflection or of reflections of any element behind the microphone such as the piano wires and the mounting elements.

In spite of this, the time-selective technique proved to be useful in eliminating other strong reflections from elements of the setup, such as a grid-floor and the flat platform below the microphones that was used to place the angular divider. An illustration of how large the influence of such reflections for LSI microphones was is shown in Fig. 6. Figure 6(a) shows the difference between the "cleaned" and "raw" frequency responses for a measurement at $\theta=0^{\circ}$. It can be seen that at such an angle the reflections from fixed elements of the measurement rig (platform, hanging structures, etc.) are significant, up to $1 \mathrm{~dB}$ below $5 \mathrm{kHz}$, while the elements behind the microphone have a small influence. Figure 6(b) shows the same results for a measurement at $\theta=135^{\circ}$. It is clear in this figure that the elements behind the microphone now have a significant influence. 
(a) $\theta=0^{\circ}$
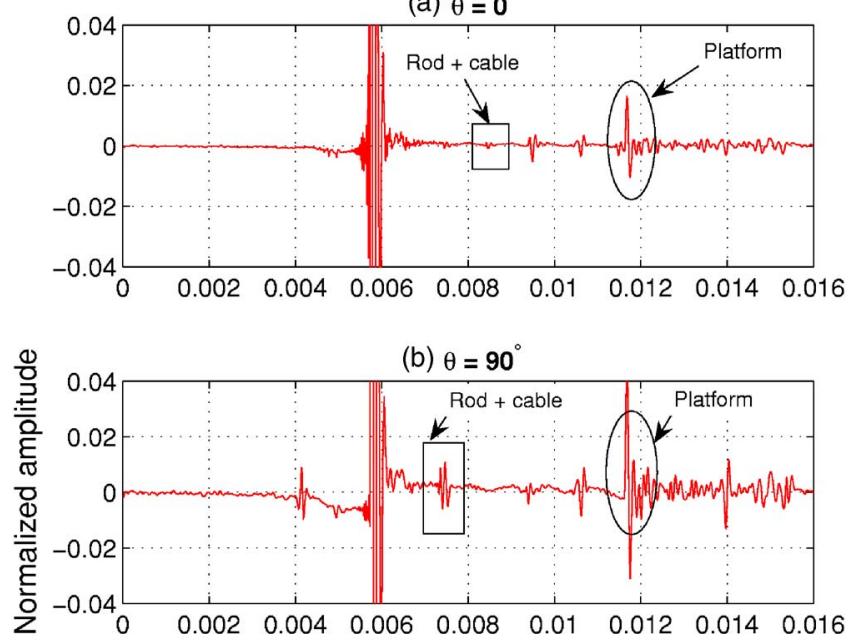

(c) $\theta=135^{\circ}$

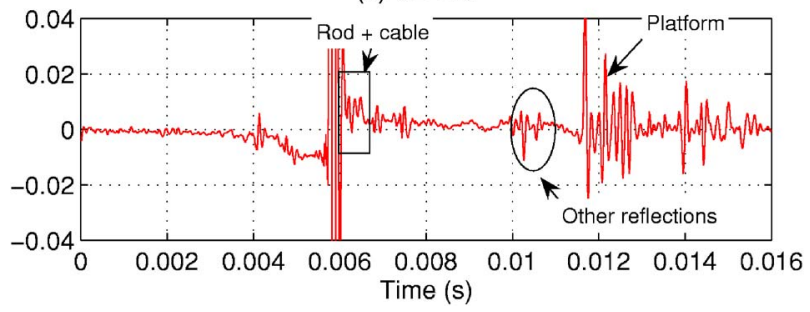

FIG. 5. Impulse response of the modified rig at different angles of incidence.

\section{THE INFLUENCE OF THE ANGULAR RESOLUTION}

The use of Eq. (2) would yield the exact value of the directivity factor. However, such an expression cannot be used in the case of the microphones. Instead, the directivity factor must be obtained using the approximation given by Eq. (3). Therefore, the influence of the angular step on the final estimate of the quantity sensitivity should be analyzed.

A suitable procedure would consist in determining the directivity factor using different angular resolutions and examining whether the estimate converges to a unique value as the resolution gets finer. Although it is intuitively evident that the finer the resolution the better the result, there is no actual indication of how accurate the estimate determined from the finest resolution will be. On the other hand, Eq. (1) can be implemented for a case where the analytical solution of the scattering problem exists, such as the case of a sphere. Once this estimate is obtained it can be compared with the outcome of the implementation of Eq. (3) for the same case. The analytical and the discrete estimates are analyzed in the following.

\section{A. The case of a sphere}

If harmonic variation with the $\exp (-i \omega t)$ sign convention is assumed, the sound pressure scattered by a solid sphere of radius $a$, centered at the origin of a spherical coordinate system, can be calculated from ${ }^{12}$
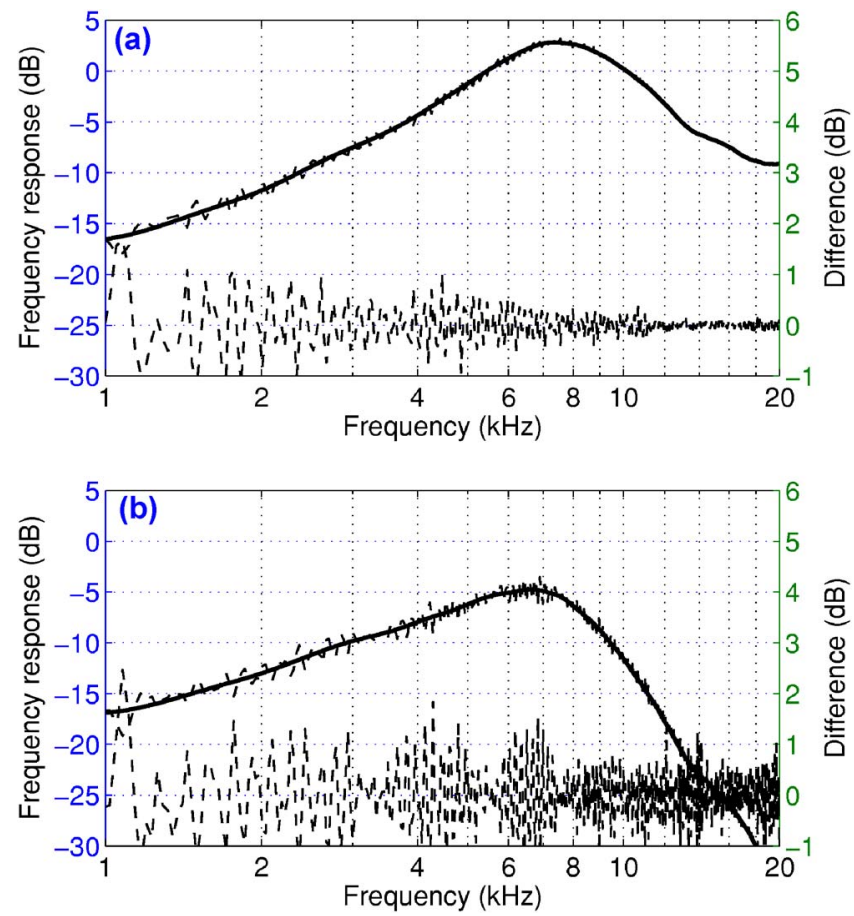

FIG. 6. Illustration of the effect of the cleaning technique: (a) Measurement of the frequency response at $\theta=0^{\circ}$, and (b) measurement of the frequency response at $\theta=135^{\circ}$. In the two graphs, the solid line is the frequency response with reflections removed, the dotted line is the measured frequency response, and the dashed line is the difference between the measured and the "cleaned" frequency responses.

$$
\begin{aligned}
p_{s}(r, \theta)= & -A \sum_{m}\left\{(2 m+1) i^{m+1}\right. \\
& \left.\times \exp \left(-i \delta_{m}\right) \sin \left(\delta_{m}\right) P_{m}(\cos \theta) h_{m}(k r)\right\},
\end{aligned}
$$

where $A$ is the amplitude of an incident plane wave coming from the direction $\theta, h_{m}(x)$ is the spherical Hankel function of the first kind and order $m, \mathrm{P}_{m}$ is the Legendre function of order $m, k$ is the wave number, $r$ is the distance to the observation point which in this case is the radius of the sphere, and the angle $\delta_{m}$ is defined as

$$
\delta=\arctan \left\{\frac{(m+1) j_{m+1}(k a)-m j_{m-1}(k a)}{m n_{m-1}(k a)-(m+1) n_{m+1}(k a)}\right\},
$$

where $j_{m}$ and $n_{m}$ are spherical Bessel and Neumann functions of order $m$.

In order to determine the total sound pressure the sound pressure of the incident plane wave must be added. In spherical coordinates this is

$$
p_{i}(r, \theta)=A \sum_{m=0}^{\infty}(2 m+1) i^{m+1} \mathrm{P}_{m}(\cos \theta) j_{m}(k r) .
$$

Thus, the total pressure on the surface of the sphere is

$$
\begin{aligned}
p_{t}(r, \theta)= & A\left\{\sum _ { m = 0 } ^ { \infty } ( 2 m + 1 ) i ^ { m } \mathrm { P } _ { m } ( \operatorname { c o s } \theta ) \left[j_{m}(k a)-i\right.\right. \\
& \left.\left.\times \exp \left(-i \delta_{m}\right) \sin \left(\delta_{m}\right) j_{m}(k a)\right]\right\} .
\end{aligned}
$$

Equation (14) can be then integrated as in Eq. (1) or it can be

Barrera-Figueroa et al.: Random-incidence response of microphones 

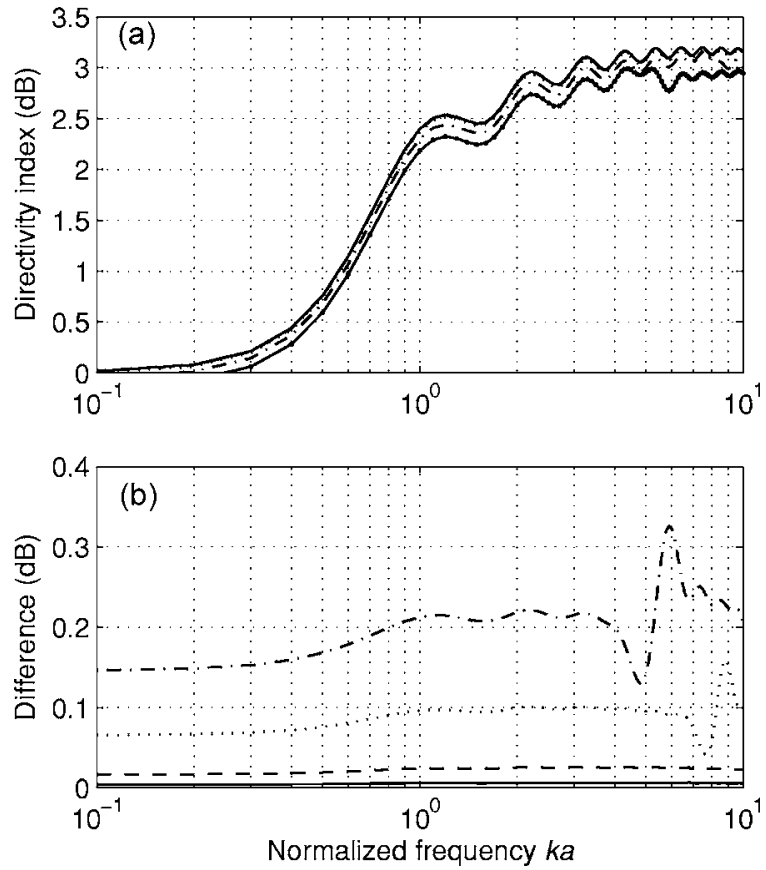

FIG. 7. (a) Directivity index obtained: analytically (solid line), and from discrete values of $\theta, 5^{\circ}$ (dashed line), $10^{\circ}$ (dotted line), $20^{\circ}$ (dashed-dotted line), and $30^{\circ}$ (solid line with dot markers). (b) Difference between the analytical and the discrete estimate of the directivity index: $5^{\circ}$ (solid line), $10^{\circ}$ (dashed line), $20^{\circ}$ (dotted line), and $30^{\circ}$ (dashed-dotted line).

estimated at discrete values of $\theta$, and then an estimate of the directivity factor can be determined using Eq. (3).

Figure 7 shows the difference between an estimate of the directivity index obtained by numerical integration and estimates obtained from the discrete summation of the function at discrete values of $\theta, 20^{\circ}, 10^{\circ}$, and $5^{\circ}$. The results are discussed further in Sec. V.

\section{B. The case of a microphone}

Unlike the case of the sphere, there is no analytical expression for the diffraction of an impinging sound wave on a microphone. Therefore, it is only possible to apply Eq. (3) to the ratio of the frequency response measured at discrete values of the angle $\theta$ to the frequency response at $\theta=0^{\circ}$. Thus, it is not possible to establish an exact reference of the directivity factor that can be compared with the discrete estimate. However, the differences between discrete estimates obtained using increasing angular steps can be compared with the case of the sphere. Figure 8 shows the difference between discrete estimates obtained using $5^{\circ}, 10^{\circ}, 20^{\circ}$, and $30^{\circ}$. The results are discussed further in Sec. V.

\section{DIRECTIVITY INDEX AND RANDOM-INCIDENCE CORRECTION}

The directivity index of LS1 and LS2 microphones has been determined experimentally. Four LS1 microphones were used in the investigation, and one of them was measured four times whereas another was measured twice. This gives a total of eight measurements. The results are shown in Fig. 9(a). Six LS2 microphones were used in the investiga-
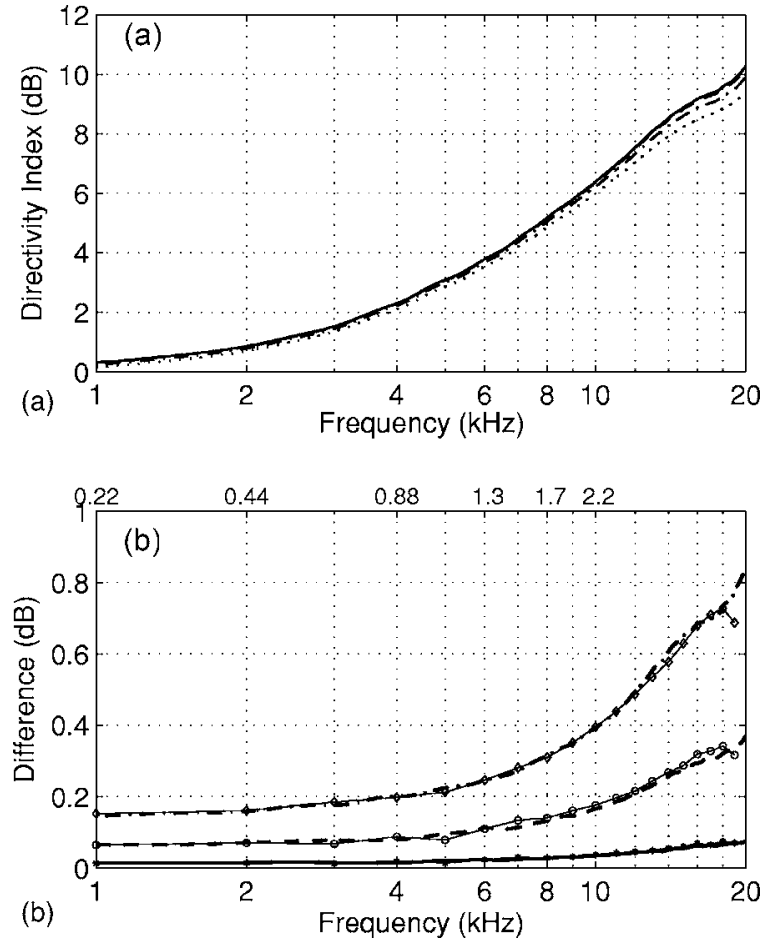

FIG. 8. (a) Directivity index of LS1 microphones obtained experimentally using discrete values of $\theta, 5^{\circ}$ (solid line), $10^{\circ}$ (dashed line), $20^{\circ}$ (dotted line), and $30^{\circ}$ (dashed-dotted line), (b) Difference between the discrete estimate of the directivity index using $5^{\circ}$ and the estimate obtained using $10^{\circ}$ (solid line), $20^{\circ}$ (dashed line), and $30^{\circ}$ (dotted line). The solid lines with open-circle, squared and closed-circle markers are the numerical estimates of the difference.

tion as well. Two of them were measured twice; this gives a total of eight measurements. The results are shown in Fig. 9(b).

Equations (5) and (6) give the possibility of estimating the random-incidence sensitivity or the random incidence correction from the directivity factor and the free-field sensitivity or the free-field correction of the microphone at $\theta$ $=0$. Figure 10 shows the random-incidence correction of LS1 and LS2 microphones obtained experimentally and numerically. Tables I and II list the values of the random-incidence correction and the standard deviation of the measurements of the directivity index. The estimate of the random-incidence correction has been obtained using Eq. (6). The free-field correction is the difference between free-field and pressure sensitivities as described in Ref. 3.

The numerical estimates were obtained using the BEM formulation described in Ref. 13. The geometry used in the simulations was a microphone mounted on an end of a cylindrical rod of the same diameter of the microphones. The semi-infinite rod was approximated by a rod of $60 \mathrm{~cm}$ long with a hemispherical back end. This will introduce a small disturbance in the simulated results because of reflections from the back of the rod. However, because of the length of the rod, these reflections are expected to have a small amplitude. The frequency range used in the calculations is from 1 to $20 \mathrm{kHz}$ for LS1 microphones and from 1 to $30 \mathrm{kHz}$ for LS2 microphones. The size of the smallest element in the axisymmetric mesh is 2.5 and $1.5 \mathrm{~mm}$ for LS1 and LS2 microphones, respectively. Thus, there will be at least 4 ele- 
(a) LS1

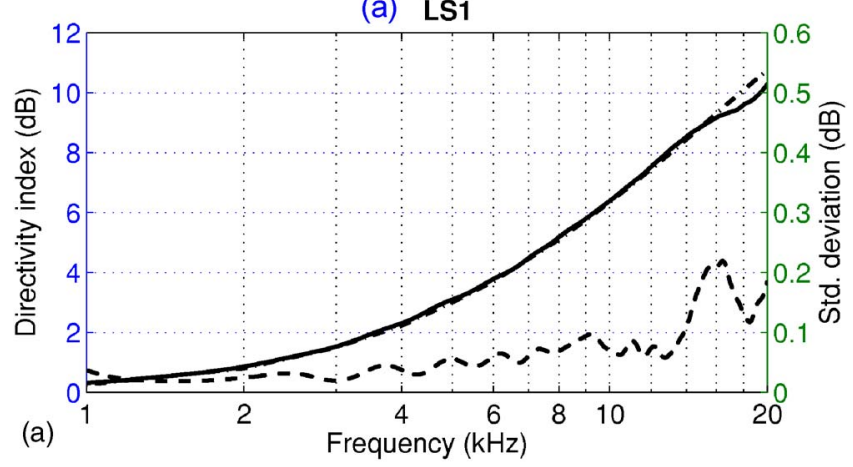

(b) LS2

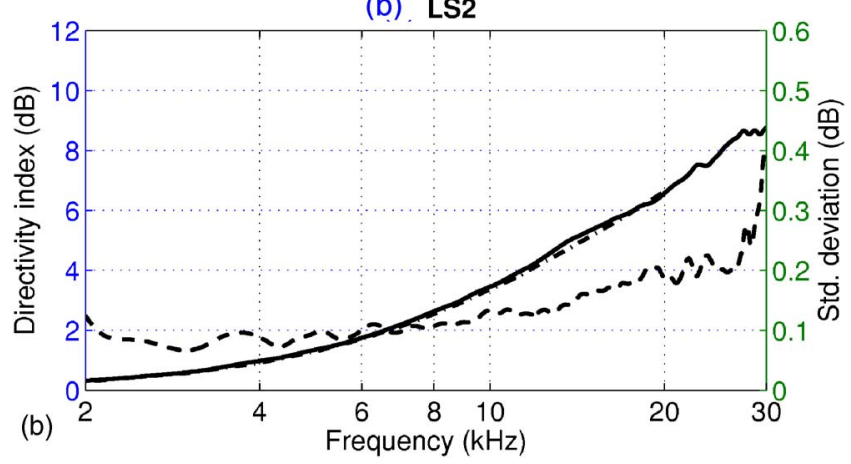

FIG. 9. Experimental directivity index of (a) LS1, and (b) LS2 microphones: Average (solid line), standard deviation (dashed line), and numerical estimate (dashed-dotted line). Standard deviation scale on right y axis.

ments per wavelength at the highest frequency. In order to avoid the nonuniqueness problem a random CHIEF point has been added in the interior of the geometry as described in Ref. 14, and the calculation have been checked by determining the condition numbers of the BEM matrices ${ }^{15}$ and by repeating calculations with small frequency shifts. In this investigation it has been assumed that the displacement of the microphone diaphragm is described by a Bessel function of the first kind. ${ }^{12,16}$

\section{DISCUSSION}

The time-selective technique is very effective in removing reflections from the measurement rig, especially those that are fully separated from the direct impulse response. The rig can be optimized for reducing the amplitude of the reflections. However, even when a time-selective technique is used some reflections will coincide with a part of the direct impulse response.

The difference between the analytical and the discrete estimates of the directivity index of the sphere is nearly constant in the whole frequency range for all angular steps, whereas the directivity index of the microphone increases with the frequency, and the slope changes as the angular resolution coarsens. The reason is that microphones are much more directional than spheres. However, the differences between the discrete estimates obtained using $5^{\circ}$ and $10^{\circ}$ are of the same order of magnitude for the microphone and the sphere, about $0.01 \mathrm{~dB}$ at low frequencies; this is also the case for the difference between $10^{\circ}$ and $30^{\circ}$, about
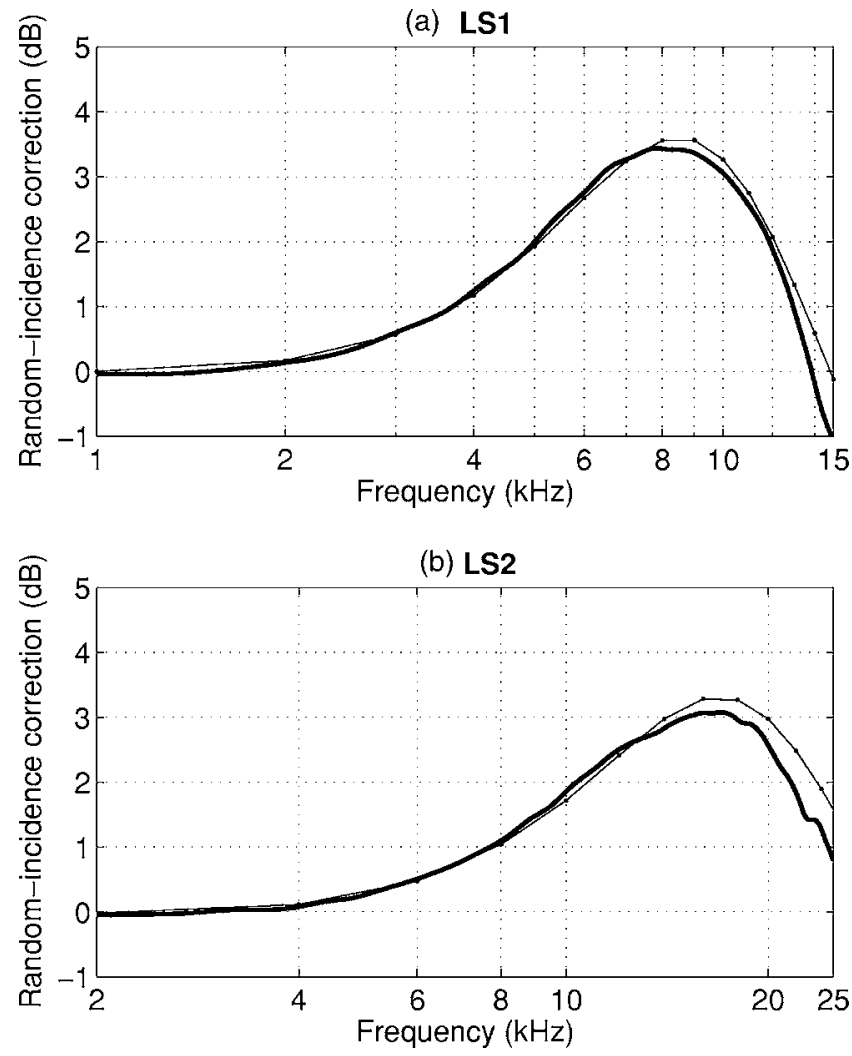

FIG. 10. Random-incidence correction of (a) LS1, and (b) LS2 microphones: Experimental (solid line), and numerical estimate (solid line with dot markers).

$0.06 \mathrm{~dB}$ at low frequencies. Thus, the difference between the analytical estimate and the discrete estimate for the sphere gives a reliable idea of the accuracy that can be reached using different angular resolutions. A resolution of $5^{\circ}$ seems to be adequate because the difference is less than $0.01 \mathrm{~dB}$; using a resolution finer than $5^{\circ}$ would improve the accuracy very little while increasing the measurement time because of the additional measurements needed. The numerical estimate of the differences confirms the experimental results.

TABLE I. Random-incidence correction and the standard deviation of the directivity index of LS1 microphones.

\begin{tabular}{lrcccc}
\hline \hline $\begin{array}{c}f \\
(\mathrm{kHz})\end{array}$ & $\begin{array}{c}C_{\mathrm{RI}} \\
(\mathrm{dB})\end{array}$ & $\begin{array}{c}s \\
(\mathrm{~dB})\end{array}$ & $\begin{array}{c}f \\
(\mathrm{kHz})\end{array}$ & $\begin{array}{c}C_{\mathrm{RI}} \\
(\mathrm{dB})\end{array}$ & $\begin{array}{c}s \\
(\mathrm{~dB})\end{array}$ \\
\hline 1.0 & -0.05 & 0.04 & 8.5 & 3.41 & 0.08 \\
1.5 & 0.00 & 0.02 & 9.0 & 3.37 & 0.09 \\
2.0 & 0.13 & 0.02 & 9.5 & 3.23 & 0.09 \\
2.5 & 0.31 & 0.03 & 10.0 & 3.06 & 0.07 \\
3.0 & 0.60 & 0.02 & 10.5 & 2.83 & 0.06 \\
3.5 & 0.86 & 0.04 & 11.0 & 2.56 & 0.09 \\
4.0 & 1.25 & 0.04 & 11.5 & 2.27 & 0.06 \\
4.5 & 1.60 & 0.04 & 12.0 & 1.88 & 0.07 \\
5.0 & 2.00 & 0.06 & 12.5 & 1.45 & 0.06 \\
5.5 & 2.43 & 0.04 & 13.0 & 0.94 & 0.06 \\
6.0 & 2.76 & 0.06 & 13.5 & 0.40 & 0.08 \\
6.5 & 3.10 & 0.05 & 14.0 & -0.17 & 0.11 \\
7.0 & 3.27 & 0.06 & 14.5 & -0.70 & 0.15 \\
7.5 & 3.40 & 0.07 & 15.0 & -1.03 & 0.19 \\
8.0 & 3.43 & 0.07 & & & \\
\hline \hline
\end{tabular}


TABLE II. Random-incidence correction and the standard deviation of the directivity index of LS2 microphones.

\begin{tabular}{|c|c|c|c|c|c|}
\hline $\begin{array}{c}f \\
(\mathrm{kHz})\end{array}$ & $\begin{array}{c}C_{\mathrm{RI}} \\
(\mathrm{dB})\end{array}$ & $\begin{array}{c}s \\
(\mathrm{~dB})\end{array}$ & $\begin{array}{c}f \\
(\mathrm{kHz})\end{array}$ & $\begin{array}{c}C_{\mathrm{RI}} \\
(\mathrm{dB})\end{array}$ & $\begin{array}{c}s \\
(\mathrm{~dB})\end{array}$ \\
\hline 2.0 & -0.05 & 0.12 & 16.5 & 3.06 & 0.17 \\
\hline 2.5 & -0.04 & 0.08 & 17.0 & 3.08 & 0.18 \\
\hline 3.0 & 0.01 & 0.07 & 17.5 & 3.05 & 0.18 \\
\hline 3.5 & 0.03 & 0.09 & 18.0 & 2.96 & 0.18 \\
\hline 4.0 & 0.08 & 0.09 & 18.5 & 2.91 & 0.20 \\
\hline 4.5 & 0.17 & 0.08 & 19.0 & 2.87 & 0.20 \\
\hline 5.0 & 0.26 & 0.10 & 19.5 & 2.75 & 0.20 \\
\hline 5.5 & 0.39 & 0.08 & 20.0 & 2.57 & 0.19 \\
\hline 6.0 & 0.51 & 0.10 & 20.5 & 2.37 & 0.19 \\
\hline 6.5 & 0.64 & 0.11 & 21.0 & 2.19 & 0.18 \\
\hline 7.0 & 0.78 & 0.10 & 21.5 & 2.05 & 0.20 \\
\hline 7.5 & 0.94 & 0.11 & 22.0 & 1.84 & 0.22 \\
\hline 8.0 & 1.10 & 0.11 & 22.5 & 1.58 & 0.20 \\
\hline 8.5 & 1.30 & 0.11 & 23.0 & 1.42 & 0.20 \\
\hline 9.0 & 1.48 & 0.11 & 23.5 & 1.41 & 0.22 \\
\hline 9.5 & 1.64 & 0.12 & 24.0 & 1.28 & 0.22 \\
\hline 10.0 & 1.85 & 0.13 & 24.5 & 1.03 & 0.20 \\
\hline 10.5 & 2.05 & 0.13 & 25.0 & 0.80 & 0.20 \\
\hline 11.0 & 2.20 & 0.13 & 25.5 & 0.55 & 0.20 \\
\hline 11.5 & 2.37 & 0.13 & 26.0 & 0.34 & 0.20 \\
\hline 12.0 & 2.51 & 0.13 & 26.5 & 0.16 & 0.20 \\
\hline 12.5 & 2.60 & 0.13 & 27.0 & -0.04 & 0.22 \\
\hline 13.0 & 2.68 & 0.13 & 27.5 & -0.24 & 0.26 \\
\hline 13.5 & 2.74 & 0.14 & 28.0 & -0.21 & 0.26 \\
\hline 14.0 & 2.83 & 0.15 & 28.5 & -0.39 & 0.26 \\
\hline 14.5 & 2.93 & 0.16 & 29.0 & -0.43 & 0.30 \\
\hline 15.0 & 2.99 & 0.16 & 29.5 & -0.52 & 0.35 \\
\hline 15.5 & 3.04 & 0.17 & 30.0 & -0.80 & 0.40 \\
\hline 16.0 & 3.07 & 0.17 & & & \\
\hline
\end{tabular}

An alternative might be to distribute the angles of incidence uniformly over a solid angle of $4 \pi$ such that their weighting is all the same. However, this will not improve the deviations observed at frequencies where the microphones are more directional because the number of angular positions close to the normal incidence is sparse compared to the number of positions around grazing incidence $\left(90^{\circ}\right)$. This would result in undersampling the area where the directivity of the microphones is most significant.

The reproducibility of the directivity index is better for LS1 microphones than for LS2 microphones. This is not unexpected because LS2 microphones have a lower sensitivity. The good behavior of LS1 microphones makes it possible to observe a significant deviation between 14 and $18 \mathrm{kHz}$, reaching a maximum of about $16 \mathrm{kHz}$. It is known that around $16 \mathrm{kHz}$ there is a resonance in the back cavity of the LS1 microphones. This resonance may be excited in different ways depending on how the oblique incidence modifies the movement of the diaphragm. Such a behavior cannot be detected in the LS2 case. The reason may be that the diaphragm of the LS2 is less compliant and more damped than the diaphragm of the LS1 microphones.

The estimation of the directivity index is a necessary step for determining the random-incidence response unless the absolute response of the microphones at any angle of incidence is available. The accuracy of the estimate of the random-incidence correction will be a combination of the accuracy of the experimental estimate of the directivity index and the accuracy of the normal-incidence response. Thus, the most accurate estimate of the random-incidence of a microphone can be obtained using the individual normal-incidence correction for a particular microphone. The agreement between the experimental and the numerical estimates of the random-incidence correction is very good at frequencies below the resonance frequency of each type of microphone. Above resonance the agreement degrades because of the assumed velocity distribution of the microphone membrane. The upper frequency is limited by the validity of the polynomial approximation used for calculating the normalincidence free-field correction $(12.5 \mathrm{kHz}$ for LS1 and $25 \mathrm{kHz}$ for LS2).

Finally, it is worth mentioning that the procedure for measuring the response of an axially symmetrical microphone can easily be extended to cover the case of microphones with no axial symmetry. In such a case, a discretized version of Eq. (1) should be used instead.

\section{CONCLUSIONS}

The directivity index and the random-incidence correction of laboratory standard microphones have been determined experimentally. Unwanted reflections from the measurement rig were removed from the frequency responses using a FFT-based, time-selective procedure.

The influence of the angular resolution has been studied by determining the directivity index using different angular resolutions. The case of the diffraction of the microphone was compared with the case of the diffraction of a sphere. The comparison showed that a resolution of $5^{\circ}$ represents a good compromise between accuracy and measurement time.

The accuracy of the random-incidence response depends on the accuracy of the estimate of the directivity index and the estimate of the normal-incidence free-field response. Although standardized values can be used, the lowest uncertainty is obtained when individual values of the free-field correction having a lower uncertainty than the standardized values are used for a particular microphone.

${ }^{1}$ IEC International Standard 61094-2, "Measurement microphones Part 2: Primary method for pressure calibration of laboratory standard microphones by the reciprocity technique" (International Electrotechnical Commission, Geneva, 1995).

${ }^{2}$ IEC International Standard 61094-3, "Measurement microphone Part 3: Primary method for free-field calibration of laboratory standard microphones by the reciprocity technique" (International Electrotechnical Commission, Geneva, 1995).

${ }^{3}$ IEC Technical Specification 61094-7, "Measurement microphones Part 7: Values for the difference between free-field and pressure sensitivity levels of laboratory standard microphones" (International Electrotechnical Commission, Geneva, 2006).

${ }^{4}$ C. Guianvarc'h, J.-N. Durocher, M. Bruneau, and A.-M. Bruneau, "Improved formulation of the acoustic transfer admittance of cylindrical cavities," Acust. Acta Acust. 92, 345-354 (2006).

${ }^{5}$ R. A. Kosobrodov and S. V. Kuznetsov, "Acoustic transfer impedance of plane-wave coupler," Acust. Acta Acust. 92, 513-520 (2006).

${ }^{6}$ S. Barrera-Figueroa, K. Rasmussen, and F. Jacobsen, "On experimental determination of the free-field correction of Laboratory Standard microphones at normal incidence," Metrologia 44, 57-63 (2007).

7IEC International Standard 61183, "Random incidence and diffuse-field calibration of sound level meters" (International Electrotechnical Commis- 
sion, Geneva, 1994).

${ }^{8}$ L. E. Kinsler, A. R. Frey, A. B. Copens, and J. V. Sanders, Fundamentals of Acoustics (Wiley, New York, 2000).

${ }^{9}$ L. L. Beranek, Acoustical Measurements (Acoustical Society of America, Melville, 1988).

${ }^{10} \mathrm{~S}$. Barrera-Figueroa, K. Rasmussen, and F. Jacobsen, "A time-selective technique for free-field reciprocity calibration of condenser microphones," J. Acoust. Soc. Am. 114, 1467-1476 (2003).

${ }^{11}$ R. Heyser, "Acoustical measurements by time delay spectrometry," J. Audio Eng. Soc. 15, 370-382 (1967).

${ }^{12}$ P. M. Morse and K. U. Ingard, Theoretical Acoustics (McGraw-Hill, New
York, 1968/1984).

${ }^{13} \mathrm{P}$. M. Juhl, "A numerical investigation of standard condenser microphones," J. Sound Vib. 177, 433-446 (1994).

${ }^{14}$ H. A. Schenck, "Improved integral formulation for acoustic radiation problems," J. Acoust. Soc. Am. 44, 45-58 (1968).

${ }^{15}$ P. M. Juhl, F. Jacobsen, V. Cutanda-Henríquez, and S. Quirós-Alpera, "On the non-uniqueness problem in a 2-D half-space BEM formulation," in Proceedings of Ninth International Congress on Sound and Vibration, Orlando, FL (2002), CD-ROM.

${ }^{16}$ A. J. Zuckerwar, "Theoretical response of condenser microphones," J. Acoust. Soc. Am. 64, 1278-1285 (1978). 